\section{OPEN ACCESS}

Edited and reviewed by: George Qian Li, Western Sydney University, Australia

*Correspondence: Xue-Qi Fu

fxq@jlu.edu.cn

Xuan-Jun Wang

wangxuanjun@gmail.com Jun Sheng

shengj@ynau.edu.cn

${ }^{\dagger}$ These authors have contributed equally to this work

Specialty section: This article was submitted to

Ethnopharmacology, a section of the journal Frontiers in Pharmacology

Received: 10 January 2020 Accepted: 15 April 2020 Published: 30 April 2020

Citation:

Wang Q, Zi C-T, Wang J, Wang Y-N, Huang $Y-W, F u X-Q$, Wang $X-J$ and

Sheng J (2020) Corrigendum: Dendrobium officinale Orchid Extract

Prevents Ovariectomy-Induced

Osteoporosis In Vivo and Inhibits RANKL-Induced Osteoclast

Differentiation In Vitro.

Front. Pharmacol. 11:578.

doi: 10.3389/fphar.2020.00578

\title{
Corrigendum: Dendrobium officinale Orchid Extract Prevents Ovariectomy-Induced Osteoporosis In Vivo and Inhibits RANKL-Induced Osteoclast Differentiation In Vitro
}

\begin{abstract}
Qi Wang ${ }^{1,2,3 \dagger}$, Cheng-Ting Zi ${ }^{1,2,3 \dagger}$, Jing Wang ${ }^{1,2,4}$, Yu-Na Wang ${ }^{1,2,4}$, Ye-Wei Huang ${ }^{1,2,4}$, Xue-Qi Fu ${ }^{5 *}$, Xuan-Jun Wang ${ }^{1,2,3,6 *}$ and Jun Sheng ${ }^{1,2,6 *}$

${ }^{1}$ Key Laboratory of Pu-erh Tea Science, Ministry of Education, Yunnan Agricultural University, Kunming, China, ${ }^{2}$ Tea Research Center of Yunnan, Kunming, China, ${ }^{3}$ College of Tea Science, Yunnan Agricultural University, Kunming, China, ${ }^{4}$ College of Food Science and Technology, Yunnan Agricultural University, Kunming, China, ${ }^{5}$ College of Life Sciences, Jilin University, Changchun, China, ${ }^{6}$ State Key Laboratory for Conservation and Utilization of Bio-Resources in Yunnan, Kunming, China
\end{abstract}

Keywords: DOE, postmenopausal osteoporosis, ovariectomy, bone quality, osteoclastogenesis

\section{A Corrigendum on}

Dendrobium officinale Orchid Extract Prevents Ovariectomy-Induced Osteoporosis in Vivo and Inhibits RANKL-Induced Osteoclast Differentiation in Vitro

by Wang Q, Zi C-T, Wang J, Wang Y-N, Huang Y-W, Fu X-Q, Wang X-J and Sheng J (2018). Front. Pharmacol. 8:966. doi: 10.3389/fphar.2017.00966

In the original article, there was a mistake in Figure $\mathbf{4}$ as published. Panels 2 and 3 (cortical bone tissue stained with H\&E for OVX Model and XLGB treated group, respectively) of Figure 4D in the original article was the same images as panels 2 and 3 of Figure $2 \mathrm{E}$ in Liang $Q, L v M$, Zhang $X, H u J$, Wu Y, Huang Y, Wang X and Sheng J (2018) Effect of Black Tea Extract and Thearubigins on Osteoporosis in Rats and Osteoclast Formation in vitro. Front. Physiol. 9:1225. doi: 10.3389/fphys. 2018.01225. Based on the 3R (Reduction, Replacement, and Refinement) principle of experimental animals, the authors simultaneously and systematically evaluated the pharmacological effects of Dendrobium officinale Orchid extract, black tea extract, and thearubigins in preventing osteoporosis using the same batch of ovariectomized (OVX) female rats as the animal model of postmenopausal osteoporosis in the animal experiment study. They collected the data and published two articles and accidentally reused the same images in them. The corrected Figure $\mathbf{4}$ appears below.

The authors apologize for this error and state that this does not change the scientific conclusions of the article in any way. The original article has been updated.

Copyright $\odot 2020$ Wang, Zi, Wang, Wang, Huang, Fu, Wang and Sheng. This is an open-access article distributed under the terms of the Creative Commons Attribution License (CC BY). The use, distribution or reproduction in other forums is permitted, provided the original author(s) and the copyright owner(s) are credited and that the original publication in this journal is cited, in accordance with accepted academic practice. No use, distribution or reproduction is permitted which does not comply with these terms. 

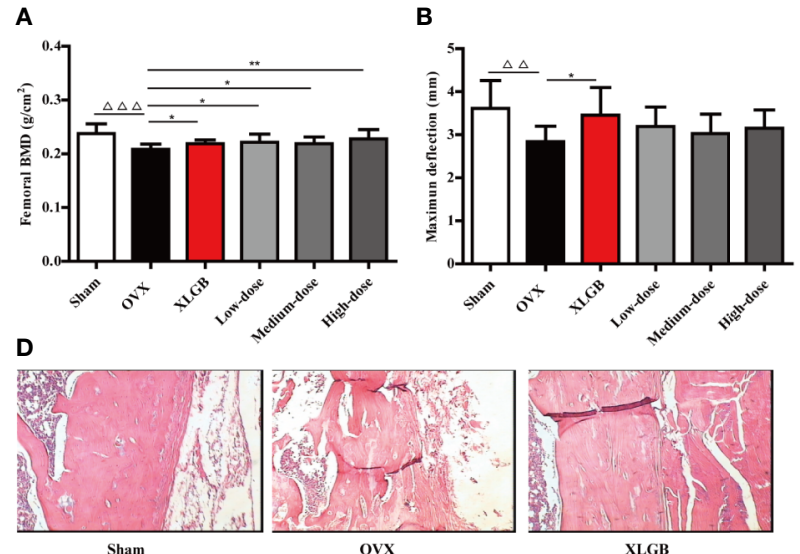

Sham

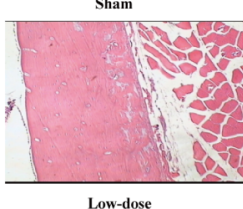

F

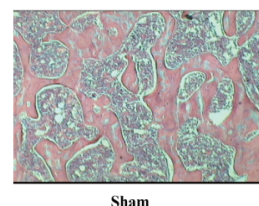

Sham

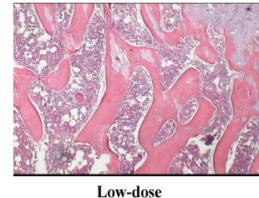

Low-dose ovx

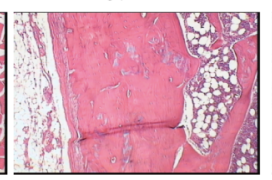

Medium-dose

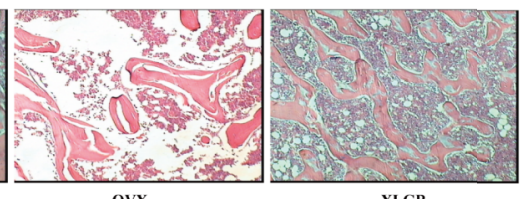

ovx

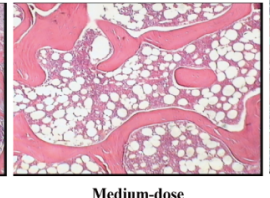

Medium-dose

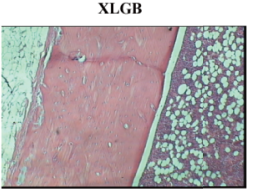

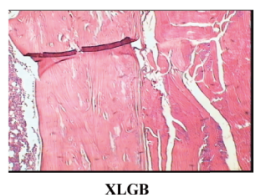

C

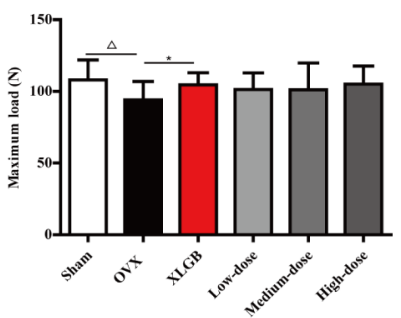

E

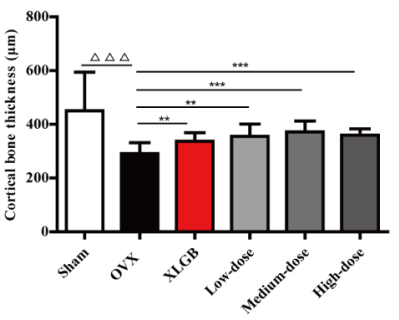

G

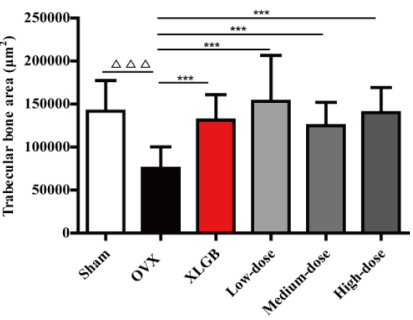

FIGURE 4 | DOE treatment improves bone quality in OVX rats: (A) femoral BMD; (B) maximum deflection; (C) maximum load; (D) cortical bone tissue stained with H\&E; (E) calculated cortical bone thickness; (F) trabecular bone tissue stained with H\&E; and (G) calculated trabecular bone area. Representative images were acquired using a medical image analysis system at an original magnification of $\times 400$. All data are presented as means \pm SEM ( $n=10$ ). $\triangle P<0.05, \triangle \triangle P<0.01$, and $\triangle \triangle \triangle P<0.001$ versus the sham group, and ${ }^{*} P<0.05$, ${ }^{* *} P<0.01$, and ${ }^{* * *} P<0.001$ versus the OVX group. 